\title{
ITERATIVE APPROXIMATION OF A SOLUTION OF A GENERAL VARIATIONAL-LIKE INCLUSION IN BANACH SPACES
}

\author{
C. E. CHIDUME, K. R. KAZMI, and H. ZEGEYE
}

Received 29 September 2002

\begin{abstract}
We introduce a class of $\eta$-accretive mappings in a real Banach space and show that the $\eta$-proximal point mapping for $\eta$ - $m$-accretive mapping is Lipschitz continuous. Further, we develop an iterative algorithm for a class of general variational-like inclusions involving $\eta$ accretive mappings in real Banach space, and discuss its convergence criteria. The class of $\eta$-accretive mappings includes several important classes of operators that have been studied by various authors.
\end{abstract}

2000 Mathematics Subject Classification: 47H04, 47H06, 47H10, 47J20, 47J25, 47J30, 49J40.

1. Introduction. Variational inequality theory has emerged as a powerful tool for a wide class of unrelated problems arising in various branches of physical, engineering, pure, and applied sciences in a unified and general framework (see, e.g., [5, 6, 7]). Variational inequalities have been extended and generalized in different directions by using novel and innovative techniques and ideas, both for their own sake and for their applications. An important and useful generalization of variational(-like) and quasivariational(-like) inequalities is a variational(-like) inclusion.

In recent years, much attention has been given to developing efficient and implementable numerical methods including projection method and its variant forms, linear approximation, auxiliary principle method, and descent and Newton's methods. In 1994, Hassouni and Moudafi [8] introduced and studied a class of variational inclusions and developed a perturbed iterative algorithm for the variational inclusions. Adly [1], Huang [9], Kazmi [11], Ding [2] have obtained some important extensions of the result in $[8]$.

Recently, Ding and Luo [4] and Ding [3] introduced the concepts of $\eta$-subdifferential and $\eta$-proximal point mapping of a proper function and developed some perturbed iterative algorithms for some classes of variational-like inclusions.

Motivated by recent works going on in this direction, we define various types of $\eta$ accretive mappings in real Banach space and establish some properties of $\eta$-proximal point mappings for $\eta$ - $m$-accretive mappings. Further, we consider a class of general variational-like inclusions in real Banach spaces and develop iterative algorithms for this class of inclusions by making use of $\eta$-proximal point mapping. The convergence analysis for the developed iterative algorithms is also studied. The results presented in this paper extend and improve many known results in the literature.

2. $\eta$ - $m$-accretive mappings. Throughout this paper, we assume that $E$ is a real Banach space equipped with norm $\|\cdot\| ; E^{*}$ is the topological dual space of $E$ equipped 
with norm $|\|\cdot\|| \mathrm{CB}(E)$ is the family of all nonempty closed and bounded subsets of $E ; 2^{E}$ is a power set of $E ; H(\cdot, \cdot)$ is the Hausdorff metric on $\mathrm{CB}(E)$ defined by

$$
H(A, B)=\max \left\{\sup _{x \in A} \inf _{y \in B} d(x, y), \sup _{y \in B} \inf _{x \in A} d(x, y)\right\} ;
$$

$\langle\cdot, \cdot\rangle$ is the dual pair between $E$ and $E^{*}$, and $J: E \rightarrow 2^{E^{*}}$ is the normalized duality mapping defined by

$$
J(x)=\left\{f \in E^{*}:\langle x, f\rangle=\|x\|^{2},\|x\|=|\|f\||\right\}, \quad x \in E .
$$

We observe immediately that if $E=H$, a Hilbert space, then $J$ is the identity map on $H$.

First, we introduce the following definitions.

DEFINITION 2.1. A mapping $\eta: E \times E \rightarrow E$ is said to be

(i) accretive, if there exists $j \eta(u, v) \in J(\eta(u, v))$ such that

$$
\langle u-v, j \eta(u, v)\rangle \geq 0, \quad \forall u, v \in E
$$

(ii) strictly accretive, if there exists $j \eta(u, v) \in J(\eta(u, v))$ such that

$$
\langle u-v, j \eta(u, v)\rangle \geq 0, \quad \forall u, v \in E,
$$

and equality holds if and only if $u=v$;

(iii) $\delta$-strongly accretive, if there exist $j \eta(u, v) \in J(\eta(u, v))$ and $\delta>0$ such that

$$
\langle u-v, j \eta(u, v)\rangle \geq \delta\|u-v\|^{2}, \quad \forall u, v \in E
$$

(iv) $\tau$-Lipschitz continuous, if there exist $j \eta(u, v) \in J(\eta(u, v))$ and $\tau>0$ such that

$$
|\|j \eta(u, v)|| \mid \leq \tau\| u-v \|, \quad \forall u, v \in E .
$$

DEFINITION 2.2. Let $\eta: E \times E \rightarrow E$ be a single-valued mapping. Then a multivalued mapping $M: E \rightarrow 2^{E}$ is said to be

(i) $\eta$-accretive, if there exists $j \eta(u, v) \in J(\eta(u, v))$ such that

$$
\langle x-y, j \eta(u, v)\rangle \geq 0, \quad \forall u, v \in E, \forall x \in M u, y \in M v
$$

(ii) strictly $\eta$-accretive, if there exists $j \eta(u, v) \in J(\eta(u, v))$ such that

$$
\langle x-y, j \eta(u, v)\rangle \geq 0, \quad \forall u, v \in E, \forall x \in M u, y \in M v,
$$

and equality holds if and only if $u=v$;

(iii) $\gamma$-strongly $\eta$-accretive, if there exist $j \eta(u, v) \in J(\eta(u, v))$ and $\gamma>0$ such that

$$
\langle x-y, j \eta(u, v)\rangle \geq \gamma\|u-v\|^{2}, \quad \forall u, v \in E, \forall x \in M u, y \in M v
$$

(iv) $\eta$-m-accretive, if $M$ is $\eta$-accretive and $(I+\rho M)(E)=E$ for any $\rho>0$, where $I$ stands for identity mapping. 
REMARK 2.3. In Definition 2.2, if $\eta(u, v)=u-v$, for all $u, v \in E$, we recover the usual definitions of accretiveness of the multivalued mapping $E$.

Definition 2.4. A multivalued mapping $M: E \rightarrow 2^{E}$ is said to be $\alpha$-H-Lipschitz continuous, if there exists $\alpha>0$ such that

$$
H(M u, M v) \leq \alpha\|u-v\|, \quad \forall u, v \in E
$$

We need the following lemma in the sequel.

LEMMA 2.5 [13]. Let $E$ be a real Banach space and $J: E \rightarrow 2^{E^{*}}$ the normalized duality mapping. Then for any $x, y \in E$,

$$
\|x+y\|^{2} \leq\|x\|^{2}+2\langle y, j(x+y)\rangle, \quad \forall j(x+y) \in J(x+y) .
$$

Now, we prove the following lemmas.

LEMMA 2.6. Let $\eta: E \times E \rightarrow E$ be a strictly accretive mapping and let $M: E \rightarrow 2^{E}$ be $\eta$-m-accretive multivalued mapping. Then

(a) $\langle x-y, j \eta(u, v)\rangle \geq 0$, for all $(y, v) \in \operatorname{Graph}(M)$, implies $(x, u) \in \operatorname{Graph}(M)$, where $\operatorname{Graph}(M):=\{(x, u) \in E \times E: x \in M u\}$;

(b) the mapping $(I+\rho M)^{-1}$ is single-valued for all $\rho>0$.

Proof. (a) Suppose, on the contrary, that there exists $\left(x_{0}, u_{0}\right) \notin \operatorname{Graph}(M)$ such that

$$
\left\langle x_{0}-y, j \eta\left(u_{0}, v\right)\right\rangle \geq 0, \quad \forall(y, v) \in \operatorname{Graph}(M) .
$$

Since $M$ is $\eta$ - $m$-accretive, we have $(I+\rho M)(E)=E$, and hence there exists $\left(x_{1}, u_{1}\right) \in$ $\operatorname{Graph}(M)$ such that

$$
x_{1}+\rho u_{1}=x_{0}+\rho u_{0} .
$$

Now, first set $(y, v)=\left(x_{1}, u_{1}\right)$ in (2.12), and then, from the resultant inequality and (2.13), we obtain

$$
0 \leq\left\langle x_{0}-x_{1}, j \eta\left(u_{0}, u_{1}\right)\right\rangle=\rho\left\langle u_{1}-u_{0}, j \eta\left(u_{0}, u_{1}\right)\right\rangle,
$$

which implies that

$$
\rho\left\langle u_{0}-u_{1}, j \eta\left(u_{0}, u_{1}\right)\right\rangle \leq 0, \quad \text { since } \rho>0 \text {. }
$$

But $\eta$ is strictly accretive, so we have

$$
0 \leq\left\langle u_{0}-u_{1}, j \eta\left(u_{0}, u_{1}\right)\right\rangle \leq 0
$$

which yields $u_{0}=u_{1}$, and hence, from (2.13), we get $x_{1}=x_{0}$, a contradiction. This completes the proof of (a). 
(b) For any given $z \in E$ and a constant $\rho>0$, let $u, v \in(I+\rho M)^{-1}(z)$. Then $\rho^{-1}(z-$ $u) \in M u$ and $\rho^{-1}(z-v) \in M v$. Now,

$$
\begin{aligned}
0 & =\rho\left\langle\rho^{-1}(z-u)-\rho^{-1}(z-v), j \eta(u, v)\right\rangle+\langle u-v, j \eta(u, v)\rangle \\
& \geq\langle u-v, j \eta(u, v)\rangle,
\end{aligned}
$$

using $\eta$-accretiveness of $M$. Since $\eta$ is strictly accretive, from the above inequality, we have $u=v$. This implies that $(I+\rho M)^{-1}$ is single valued. This completes the proof.

REMARK 2.7. By Lemma 2.6, we can define $\eta$-proximal point mapping for an $\eta$ - $m$ accretive mapping $M$ as follows:

$$
J_{\rho}^{M}(z)=(I+\rho M)^{-1}(z), \quad \forall z \in E,
$$

where $\rho>0$ is a constant and $\eta: E \times E \rightarrow E$ is a strictly accretive mapping.

LEMMA 2.8. Let $\eta: E \times E \rightarrow E$ be a $\delta$-strongly accretive and $\tau$-Lipschitz continuous mapping and let $M: E \rightarrow 2^{E}$ be an $\eta$-m-accretive mapping. Then the $\eta$-proximal point mapping $J_{\rho}^{M}$ is $\tau / \delta$-Lipschitz continuous, that is,

$$
\left\|J_{\rho}^{M}(u)-J_{\rho}^{M}(v)\right\| \leq \frac{\tau}{\delta}\|u-v\|, \quad \forall u, v \in E .
$$

Proof. Let $u, v \in E$. From the definition of $J_{\rho}^{M}$, we have $J_{\rho}^{M}(u)=(I+\rho M)^{-1}(u)$. This implies that

$$
\rho^{-1}\left(u-J_{\rho}^{M}(u)\right) \in M\left(J_{\rho}^{M}(u)\right)
$$

Similarly, we have

$$
\rho^{-1}\left(v-J_{\rho}^{M}(v)\right) \in M\left(J_{\rho}^{M}(v)\right)
$$

Since $M$ is $\eta$-accretive, we obtain

$$
\begin{aligned}
0 & \leq \rho^{-1}\left\langle\left(u-J_{\rho}^{M}(u)\right)-\left(v-J_{\rho}^{M}(v)\right), j \eta\left(J_{\rho}^{M}(u), J_{\rho}^{M}(v)\right)\right\rangle \\
& =\rho^{-1}\left\langle u-v, j \eta\left(J_{\rho}^{M}(u), J_{\rho}^{M}(v)\right)\right\rangle-\rho^{-1}\left\langle J_{\rho}^{M}(u)-J_{\rho}^{M}(v), j \eta\left(J_{\rho}^{M}(u), J_{\rho}^{M}(v)\right)\right\rangle .
\end{aligned}
$$

Since $\rho>0$, and $\eta$ is $\delta$-strongly accretive and $\tau$-Lipschitz continuous, from the above inequality, we have

$$
\delta\left\|J_{\rho}^{M}(u)-J_{\rho}^{M}(v)\right\|^{2} \leq \tau\|u-v\| \cdot\left\|J_{\rho}^{M}(u)-J_{\rho}^{M}(v)\right\| .
$$

This implies that

$$
\left\|J_{\rho}^{M}(u)-J_{\rho}^{M}(v)\right\| \leq \frac{T}{\delta}\|u-v\|, \quad \forall u, v \in E,
$$

and this completes the proof. 
3. General variational-like inclusions and iterative algorithm. Let $\eta, N: E \times E \rightarrow E$ and $G: E \rightarrow E$ be three single-valued mappings, let $S, T: E \rightarrow \mathrm{CB}(E)$ be two multivalued mappings, and let $M: E \times E \rightarrow 2^{E}$ be a multivalued mapping such that, for each $u \in E$, $M(\cdot, u)$ is $\eta$ - $m$-accretive. We consider the following general variational-like inclusion problem (in short, GVIP).

Find $u \in E, x \in S u$, and $y \in T u$ such that

$$
0 \in N(x, y)+M(G u, u)
$$

The following are special cases of GVIP (3.1).

(I) If $E=H$, a real Hilbert space, $M(\cdot, u)=\partial \phi(\cdot, u)$, where $\phi: H \times H \rightarrow \mathbb{R} \bigcup\{+\infty\}$ is such that $\phi(\cdot, u)$ is a proper and lower semicontinuous functional for all $u \in H$, and $\partial \phi(\cdot, u)$ denotes the $\eta$-subdifferential of $\phi(\cdot, u)$, then GVIP (3.1) reduces to variationallike inequality problem of finding $u \in H, x \in S u$, and $y \in T u$, such that

$$
\langle N(x, y), \eta(v, G u)\rangle \geq \phi(G u, u)-\phi(G u, v), \quad \forall v \in H
$$

similar to the problem considered by Ding [3].

(II) In inequality (3.2), if $N(x, y)=x-y$, for all $x, y \in H$, and if $S$ and $T$ are singlevalued mappings, still in Hilbert spaces, then problem (3.2) reduces to variational-like inequality problem considered by Ding and Luo [4].

We remark that for suitable choices of $N, \eta, M, S, T$, and $G$, GVIP (3.1) reduces to various classes of variational inclusions and variational inequalities (e.g., $[2,8,9,11]$ ) studied by various authors in Hilbert spaces. Our problem (3.1) is also set in more general real Banach spaces.

The following lemma, which will be used in the sequel, is an immediate consequence of the definition of $J_{\rho}^{M(\cdot, u)}$.

LEMMA 3.1. For given $u \in E, x \in S u$, and $y \in T u,(u, x, y)$ is a solution of GVIP (3.1) if and only if it is a solution of

$$
G u=J_{\rho}^{M(\cdot, u)}(G u-\rho N(x, y)),
$$

where $J_{\rho}^{M(\cdot, u)}=(I+\rho M(\cdot, u))^{-1}$ and $\rho>0$ is a constant.

Using Lemma 3.1 and a theorem of Nadler [12], we develop an iterative algorithm for finding the approximate solution of GVIP (3.1) as follows.

ITERATIVE ALGORITHM 3.2. Let $\eta, N: E \times E \rightarrow E, G: E \rightarrow E$, and $S, T: E \rightarrow \mathrm{CB}(E)$ be such that, for each $u \in E, Q(u) \subseteq G(E)$, where $Q: E \rightarrow 2^{E}$ is a multivalued mapping defined by

$$
Q(u)=\bigcup_{x \in S u} \bigcup_{y \in T u}\left(J_{\rho}^{M(\cdot, u)}(G u-\rho N(x, y))\right),
$$

where $M: E \times E \rightarrow 2^{E}$ is a multivalued mapping such that, for each $u \in E, M(\cdot, u)$ is $\eta$ - $m$-accretive. 
For given $u_{0} \in E, x_{0} \in S u_{0}$, and $y_{0} \in T u_{0}$, let

$$
w_{0}=J_{\rho}^{M\left(\cdot, u_{0}\right)}\left(G u_{0}-\rho N\left(x_{0}, y_{0}\right)\right) \in Q\left(u_{0}\right) \subseteq G(E) .
$$

Hence there exists $u_{1} \in E$ such that $w_{0}=G\left(u_{1}\right)$. Since $x_{0} \in S u_{0} \in \mathrm{CB}(E)$ and $y_{0} \in$ $T u_{0} \in \mathrm{CB}(E)$, then, by Nadler's result [12], there exist $x_{1} \in S u_{1}$ and $y_{1} \in T u_{1}$ such that

$$
\begin{aligned}
\left\|x_{0}-x_{1}\right\| & \leq\left(1+(1+1)^{-1}\right) H\left(S u_{0}, S u_{1}\right), \\
\left\|y_{0}-y_{1}\right\| & \leq\left(1+(1+1)^{-1}\right) H\left(T u_{0}, T u_{1}\right) .
\end{aligned}
$$

Let

$$
w_{1}=J_{\rho}^{M\left(\cdot, u_{1}\right)}\left(G u_{1}-\rho N\left(x_{1}, y_{1}\right)\right) \in Q\left(u_{1}\right) \subseteq G(E) .
$$

Hence there exists $u_{2} \in E$ such that $w_{1}=G\left(u_{2}\right)$. By induction, we can define iterative sequences $\left\{u_{n}\right\},\left\{G u_{n}\right\},\left\{x_{n}\right\}$, and $\left\{y_{n}\right\}$ as follows:

$$
\begin{gathered}
G u_{n+1}=J_{\rho}^{M\left(\cdot, u_{n}\right)}\left(G u_{n}-\rho N\left(x_{n}, y_{n}\right)\right), \\
x_{n} \in S u_{n}:\left\|x_{n}-x_{n+1}\right\| \leq\left(1+(1+n)^{-1}\right) H\left(S u_{n}, S u_{n+1}\right), \\
y_{n} \in T u_{n}:\left\|y_{n}-y_{n+1}\right\| \leq\left(1+(1+n)^{-1}\right) H\left(T u_{n}, T u_{n+1}\right),
\end{gathered}
$$

where $n=0,1,2,3, \ldots$ and $\rho>0$ is a constant.

\section{Convergence analysis for Iterative Algorithm 3.2.}

THEOREM 4.1. Let $E$ be a real Banach space and let $\eta: E \times E \rightarrow E$ be $\delta$-strongly accretive and $\tau$-Lipschitz continuous. Let $S, T: E \rightarrow \mathrm{CB}(E)$, and $G: E \rightarrow E$ be $\sigma$-H-Lipschitz continuous, $k$-H-Lipschitz continuous, and $\xi$-Lipschitz continuous mappings, respectively, and let $G$ be $\nu$-strongly $\eta$-accretive mapping. Let $N: E \times E \rightarrow E$ be $\alpha$-and $\beta$-Lipschitz continuous with respect to the first and second arguments, respectively, and $\gamma$-strongly $\eta$ accretive with respect to $S$ in the first argument. Let $M: E \times E \rightarrow 2^{E}$ be such that, for each fixed $w \in E, M(\cdot, w)$ is $\eta$-m-accretive mapping, and, for each $u \in E$, let $Q(u) \subseteq G(E)$, where $Q$ is defined by (3.4). Suppose that there exist $\rho>0$ and $\lambda>0$ such that, for each $w_{1}, w_{2}, v \in E$,

$$
\begin{gathered}
\left\|J_{\rho}^{M\left(\cdot, w_{1}\right)}(v)-J_{\rho}^{M\left(\cdot, w_{2}\right)}(v)\right\| \leq \lambda\left\|w_{1}-w_{2}\right\| \\
\left|\rho-\frac{\tau^{2}[\gamma-\sigma \alpha(\xi+\tau)]-v \delta k \beta(1-l)}{\tau^{2}\left(2 \sigma^{2} \alpha^{2}-k^{2} \beta^{2}\right)}\right| \\
<\frac{\sqrt{\left[\tau^{2}(\gamma-\sigma \alpha(\xi+\tau))-v \delta k \beta(1-l)\right]^{2}-\left[2 \sigma^{2} \alpha^{2}-k^{2} \beta^{2}\right]\left[\tau^{4} \xi^{2}-v^{2} \delta^{2}(1-l)^{2}\right]}}{\tau^{2}\left(2 \sigma^{2} \alpha^{2}-k^{2} \beta^{2}\right)} \\
\tau^{2} \gamma>\tau^{2} \sigma \alpha(\xi+\tau)+v \delta k \beta(1-l)+\sqrt{\left(2 \sigma^{2} \alpha^{2}-k^{2} \beta^{2}\right)\left(\tau^{4} \xi^{2}-v^{2} \delta^{2}(1-l)^{2}\right)} \\
\tau^{2} \xi>v \delta(1-l), \quad \sqrt{2} \sigma \alpha>k \beta
\end{gathered}
$$


where $l=(\tau / v) \lambda$. Then the iterative sequences $\left\{u_{n}\right\},\left\{G u_{n}\right\},\left\{x_{n}\right\}$, and $\left\{y_{n}\right\}$ generated by Iterative Algorithm 3.2 converge strongly to $u^{*}, G u^{*}, x^{*}$, and $y^{*}$, respectively, and $\left(u^{*}, x^{*}, y^{*}\right)$ is a solution of GVIP (3.1).

Proof. From Lemma 2.8, (3.8), and inequality (4.1), we have the following estimates:

$$
\begin{aligned}
&\left\|G u_{n+2}-G u_{n+1}\right\| \\
&=\left\|J_{\rho}^{M\left(\cdot, u_{n+1}\right)}\left(G u_{n+1}-\rho N\left(x_{n+1}, y_{n+1}\right)\right)-J_{\rho}^{M\left(\cdot, u_{n}\right)}\left(G u_{n}-\rho N\left(x_{n}, y_{n}\right)\right)\right\| \\
& \leq\left\|J_{\rho}^{M\left(\cdot, u_{n+1}\right)}\left(G u_{n+1}-\rho N\left(x_{n+1}, y_{n+1}\right)\right)-J_{\rho}^{M\left(\cdot, u_{n+1}\right)}\left(G u_{n}-\rho N\left(x_{n}, y_{n}\right)\right)\right\| \\
&+\left\|J_{\rho}^{M\left(\cdot, u_{n+1}\right)}\left(G u_{n}-\rho N\left(x_{n}, y_{n}\right)\right)-J_{\rho}^{M\left(\cdot, u_{n}\right)}\left(G u_{n}-\rho N\left(x_{n}, y_{n}\right)\right)\right\| \\
& \leq \frac{\tau}{\delta}\left\|G u_{n+1}-G u_{n}-\rho\left[N\left(x_{n+1}, y_{n+1}\right)-N\left(x_{n}, y_{n}\right)\right]\right\|+\lambda\left\|u_{n+1}-u_{n}\right\| \\
& \leq \frac{\tau}{\delta}\left\|G u_{n+1}-G u_{n}-\rho\left[N\left(x_{n+1}, y_{n+1}\right)-N\left(x_{n}, y_{n+1}\right)\right]\right\| \\
&+\rho \frac{\tau}{\delta}\left\|N\left(x_{n}, y_{n+1}\right)-N\left(x_{n}, y_{n}\right)\right\|+\lambda\left\|u_{n+1}-u_{n}\right\| .
\end{aligned}
$$

Since $G$ is $v$-strongly $\eta$-accretive and $\eta$ is $\tau$-Lipschitz continuous, we have the following estimates:

$$
\begin{aligned}
\tau\left\|u_{n+1}-u_{n}\right\|\left\|\left(G u_{n+1}-G u_{n}\right)\right\| & \geq\left\langle G u_{n+1}-G u_{n}, j \eta\left(u_{n+1}, G u_{n}\right)\right\rangle \\
& \geq v\left\|u_{n+1}-u_{n}\right\|^{2}
\end{aligned}
$$

implies that

$$
\left\|u_{n+1}-u_{n}\right\| \leq \frac{\tau}{v}\left\|\left(G u_{n+1}-G u_{n}\right)\right\|
$$

From the assumption that $N$ is $\beta$-Lipschitz continuous in the second argument and $T$ is $k$ - $H$-Lipschitz continuous, we get that

$$
\left\|N\left(x_{n}, y_{n+1}\right)-N\left(x_{n}, y_{n}\right)\right\| \leq \beta\left\|y_{n+1}-y_{n}\right\| \leq \beta k\left(1+(1+n)^{-1}\right)\left\|u_{n+1}-u_{n}\right\| .
$$

Furthermore, since $N$ is $\gamma$-strongly $\eta$-accretive with respect to $S$ in the first argument and $\alpha$-Lipschitz continuous with respect to the first argument and $G$ is $\xi$-Lipschitz continuous, by using Lemma 2.5 , we obtain that

$$
\begin{aligned}
& \left\|G u_{n+1}-G u_{n}-\rho\left[N\left(x_{n+1}, y_{n+1}\right)-N\left(x_{n}, y_{n+1}\right)\right]\right\|^{2} \\
& \leq\left\|G u_{n+1}-G u_{n}\right\|^{2}-2 \rho\left\langle N\left(x_{n+1}, y_{n+1}\right)-N\left(x_{n}, y_{n+1}\right),\right. \\
& \left.\quad j\left(G u_{n+1}-G u_{n}-\rho\left[N\left(x_{n+1}, y_{n+1}\right)-N\left(x_{n}, y_{n+1}\right)\right]\right)\right\rangle \\
& \leq \xi^{2}\left\|u_{n+1}-u_{n}\right\|^{2}-2 \rho\left\langle N\left(x_{n+1}, y_{n+1}\right)-N\left(x_{n}, y_{n+1}\right), j\left(\eta\left(u_{n+1}, u_{n}\right)\right)\right\rangle \\
& -2 \rho\left\langle N\left(x_{n+1}, y_{n+1}\right)\right. \\
& \quad-N\left(x_{n}, y_{n+1}\right), j\left(G u_{n+1}-G u_{n}-\rho\left[N\left(x_{n+1}, y_{n+1}\right)-N\left(x_{n}, y_{n+1}\right)\right]\right) \\
& \left.-j\left(\eta\left(u_{n+1}, u_{n}\right)\right)\right\rangle
\end{aligned}
$$




$$
\begin{aligned}
\leq & \xi^{2}\left\|u_{n+1}-u_{n}\right\|^{2}-2 \rho \gamma\left\|u_{n+1}-u_{n}\right\|^{2}+2 \rho\left\|N\left(x_{n+1}, y_{n+1}\right)-N\left(x_{n}, y_{n+1}\right)\right\| \\
& \times\left[\left\|G u_{n+1}-G u_{n}\right\|+\rho\left\|N\left(x_{n+1}, y_{n+1}\right)-N\left(x_{n}, y_{n+1}\right)\right\|+\left\|\eta\left(u_{n+1}, u_{n}\right)\right\|\right] \\
\leq & \left(\xi^{2}-2 \rho \gamma\right)\left\|u_{n+1}-u_{n}\right\|^{2}+2 \rho \alpha\left\|x_{n+1}-x_{n}\right\|\left[(\xi+\tau)\left\|u_{n+1}-u_{n}\right\|+\rho \alpha\left\|x_{n+1}-x_{n}\right\|\right] \\
= & {\left[\xi^{2}-2 \rho \gamma+2 \rho \alpha \sigma(\xi+\tau)\left(1+(1+n)^{-1}\right)+2 \rho^{2} \alpha^{2} \sigma^{2}\left(1+(1+n)^{-1}\right)^{2}\right] } \\
& \times\left\|u_{n+1}-u_{n}\right\|^{2},
\end{aligned}
$$

where $S$ is $\sigma$-H-Lipschitz continuous.

Combining (4.5), (4.7), (4.8), and (4.9), we have the following estimates:

$$
\left\|u_{n+2}-u_{n+1}\right\| \leq \theta_{n}\left\|u_{n+1}-u_{n}\right\|
$$

where

$$
\begin{aligned}
\theta_{n}:=\frac{\tau^{2}}{\nu \delta}[ & \sqrt{\xi^{2}-2 \rho \gamma+2 \rho \alpha \sigma(\xi+\tau)\left(1+(1+n)^{-1}\right)+2 \rho^{2} \alpha^{2} \sigma^{2}\left(1+(1+n)^{-1}\right)^{2}} \\
& \left.+\rho \beta k\left(1+(1+n)^{-1}\right)\right]+\frac{\tau}{v} \lambda .
\end{aligned}
$$

Letting $n \rightarrow \infty$, we obtain that $\theta_{n} \rightarrow \theta$, where

$$
\theta:=\frac{\tau^{2}}{\nu \delta}\left[\sqrt{\xi^{2}-2 \rho \gamma+2 \rho \alpha \sigma(\xi+\tau)+2 \rho^{2} \alpha^{2} \sigma^{2}}+\rho \beta k\right]+\frac{\tau}{v} \lambda .
$$

It follows from (4.10), conditions (4.2)-(4.4) and (4.11), (4.12) that $\left\{u_{n}\right\}$ is a Cauchy sequence in $E$. So, there exists $u^{*} \in E$ such that $u_{n} \rightarrow u^{*}$ as $n \rightarrow \infty$. From Lipschitz continuity of $G$, we have that

$$
\left\|G u_{n+1}-G u_{n}\right\| \leq \xi\left\|u_{n+1}-u_{n}\right\| .
$$

Also, from (3.9), we get that

$$
\left\|x_{n}-x_{n+1}\right\| \leq\left(1+(1+n)^{-1}\right) H\left(S u_{n}, S u_{n+1}\right) \leq \sigma\left(1+(1+n)^{-1}\right)\left\|u_{n}-u_{n+1}\right\| .
$$

Since $\left\{u_{n}\right\}$ is a Cauchy sequence, it follows from (4.13) and (4.14) that $\left\{G u_{n}\right\}$ and $\left\{x_{n}\right\}$ both are also Cauchy sequences. Similarly, $\left\{y_{n}\right\}$ is a Cauchy sequence. Hence there exist $x^{*}, y^{*} \in E$ such that $G u_{n} \rightarrow G u^{*}, x_{n} \rightarrow x^{*}$, and $y_{n} \rightarrow y^{*}$ as $n \rightarrow \infty$. Furthermore,

$$
\begin{aligned}
d\left(x^{*}, S u^{*}\right) & \leq\left\|x^{*}-x_{n}\right\|+d\left(x_{n}, S u^{*}\right) \\
& \leq\left\|x^{*}-x_{n}\right\|+H\left(S u_{n}, S u^{*}\right) \\
& \leq\left\|x^{*}-x_{n}\right\|+\sigma\left\|u_{n}-u^{*}\right\| \longrightarrow 0,
\end{aligned}
$$

and hence $x^{*} \in S u^{*}$. Similarly, $y^{*} \in T u^{*}$. 
Finally, we define

$$
w^{*}:=J_{\rho}^{M\left(\cdot, u^{*}\right)}\left(G u^{*}-\rho N\left(x^{*}, y^{*}\right)\right)
$$

Now, we estimate that

$$
\begin{aligned}
\left\|G u_{n+2}-w^{*}\right\| \leq & \frac{\tau}{\delta}\left\|G u_{n+1}-G u^{*}-\rho\left[N\left(x_{n+1}, y_{n+1}\right)-N\left(x^{*}, y^{*}\right)\right]\right\| \\
& +\lambda\left\|u_{n+1}-u^{*}\right\| \\
\leq & \frac{\tau}{\delta}\left[\left\|G u_{n+1}-G u^{*}\right\|+\rho\left\|N\left(x_{n+1}, y_{n+1}\right)-N\left(x^{*}, y_{n+1}\right)\right\|\right. \\
& \left.\quad+\rho\left\|N\left(x^{*}, y_{n+1}\right)-N\left(x^{*}, y^{*}\right)\right\|\right]+\lambda\left\|u_{n+1}-u^{*}\right\| \\
\leq & \frac{\tau}{\delta}\left[\left\|G u_{n+1}-G u^{*}\right\|+\rho \alpha\left\|x_{n+1}-x^{*}\right\|+\rho \beta\left\|y_{n+1}-y^{*}\right\|\right] \\
& +\lambda\left\|u_{n+1}-u^{*}\right\| \longrightarrow 0 \text { as } n \longrightarrow \infty .
\end{aligned}
$$

Thus, by Lemma 3.1, it follows that $\left(u^{*}, x^{*}, y^{*}\right)$ is a solution of GVIP (3.1), and this completes the proof.

REMARK 4.2. It is clear that $\delta \leq \tau, v \leq \xi$, and $\gamma \leq \alpha$. Further, condition (4.2) is true for suitable values of constants, for example,

(i) $\delta=\tau=v=\xi=\gamma=\alpha=1 ; \sigma=\beta=k=\lambda=0.1$; and $\rho=0.5$;

(ii) $\delta=\nu=\gamma=\sigma=\beta=k=\rho=0.1 ; \tau=\xi=\alpha=0.2$; and $\lambda=0.01$.

REMARK 4.3. Most of the results in, for example, $[4,10]$ and the references therein, are obtained in Hilbert spaces. Our theorems extend them to the more general real Banach spaces considered in this paper.

ACKNOWLEDGMENT. The third author undertook this work when he was visiting the Abdus Salam International Centre for Theoretical Physics, Trieste, Italy, as a postdoctoral fellow.

\section{REFERENCES}

[1] S. Adly, Perturbed algorithms and sensitivity analysis for a general class of variational inclusions, J. Math. Anal. Appl. 201 (1996), no. 2, 609-630.

[2] X. P. Ding, Perturbed proximal point algorithms for generalized quasivariational inclusions, J. Math. Anal. Appl. 210 (1997), no. 1, 88-101.

[3] __ Generalized quasi-variational-like inclusions with nonconvex functionals, Appl. Math. Comput. 122 (2001), no. 3, 267-282.

[4] X. P. Ding and C. L. Luo, Perturbed proximal point algorithms for general quasi-variationallike inclusions, J. Comput. Appl. Math. 113 (2000), no. 1-2, 153-165.

[5] F. Giannessi and A. Maugeri (eds.), Variational Inequalities and Network Equilibrium Problems, Plenum Press, New York, 1995.

[6] R. Glowinski, Numerical Methods for Nonlinear Variational Problems, Springer Series in Computational Physics, Springer-Verlag, New York, 1984.

[7] R. Glowinski, J.-L. Lions, and R. Trémolières, Numerical Analysis of Variational Inequalities, Studies in Mathematics and Its Applications, vol. 8, North-Holland Publishing, Amsterdam, 1981.

[8] A. Hassouni and A. Moudafi, A perturbed algorithm for variational inclusions, J. Math. Anal. Appl. 185 (1994), no. 3, 706-712. 
[9] N.-J. Huang, Generalized nonlinear variational inclusions with noncompact valued mappings, Appl. Math. Lett. 9 (1996), no. 3, 25-29.

[10] _ A new class of generalized set-valued implicit variational inclusions in Banach spaces with an application, Comput. Math. Appl. 41 (2001), no. 7-8, 937-943.

[11] K. R. Kazmi, Mann and Ishikawa type perturbed iterative algorithms for generalized quasivariational inclusions, J. Math. Anal. Appl. 209 (1997), no. 2, 572-584.

[12] S. B. Nadler Jr., Multi-valued contraction mappings, Pacific J. Math. 30 (1969), 475-488.

[13] W. V. Petryshyn, A characterization of strict convexity of Banach spaces and other uses of duality mappings, J. Funct. Anal. 6 (1970), 282-291.

C. E. Chidume: The Abdus Salam International Centre for Theoretical Physics, 34100 Trieste, Italy

E-mail address: chidume@ictp.trieste.it

K. R. Kazmi: Department of Mathematics, Aligarh Muslim University, Aligarh 202 002, India Current address: The Abdus Salam International Centre for Theoretical Physics, 34100 Trieste, Italy

E-mail address: krkazmi0@postmark.net

H. Zegeye: The Abdus Salam International Centre for Theoretical Physics, 34100 Trieste, Italy E-mail address: habz@ictp.trieste.it 


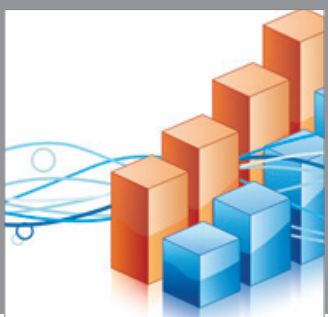

Advances in

Operations Research

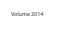

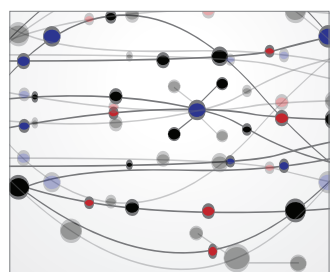

\section{The Scientific} World Journal
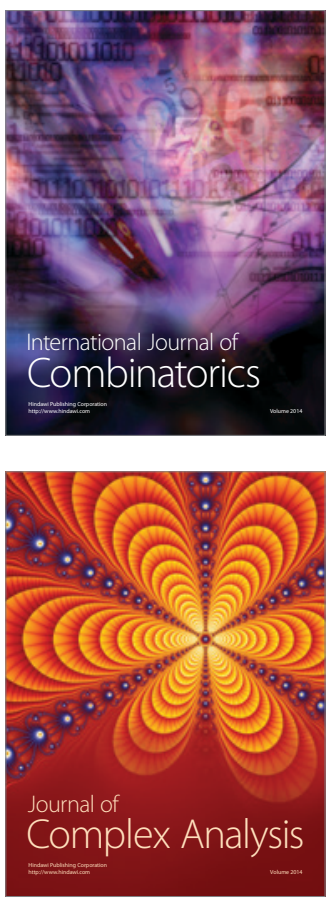

International Journal of

Mathematics and

Mathematical

Sciences
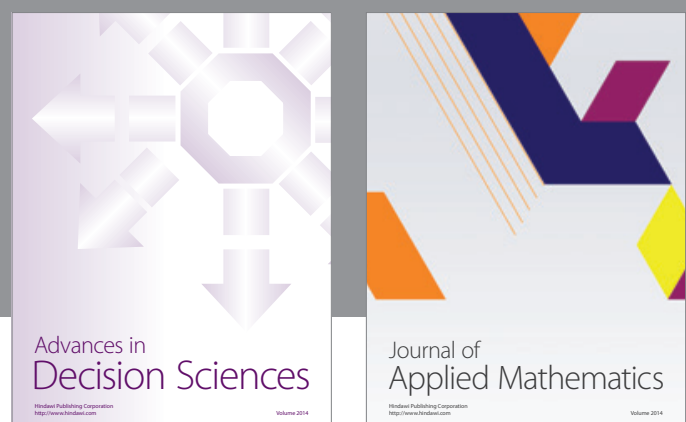

Journal of

Applied Mathematics
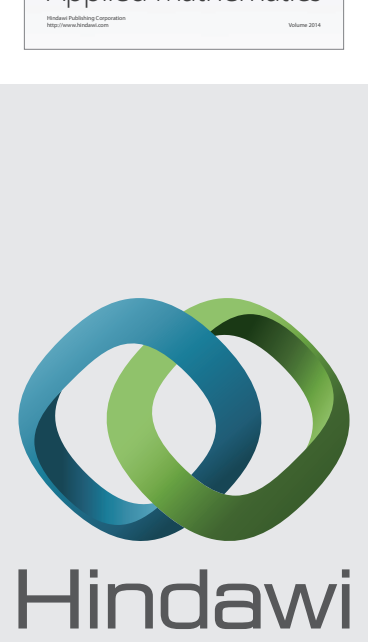

Submit your manuscripts at http://www.hindawi.com
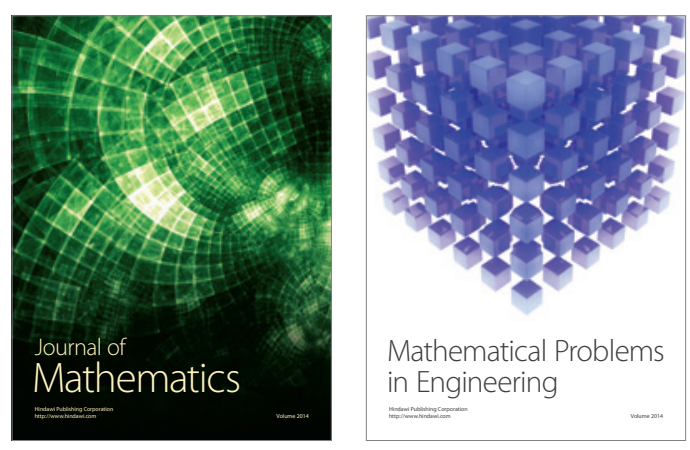

Mathematical Problems in Engineering
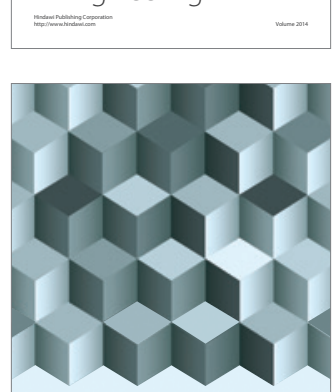

Journal of

Function Spaces
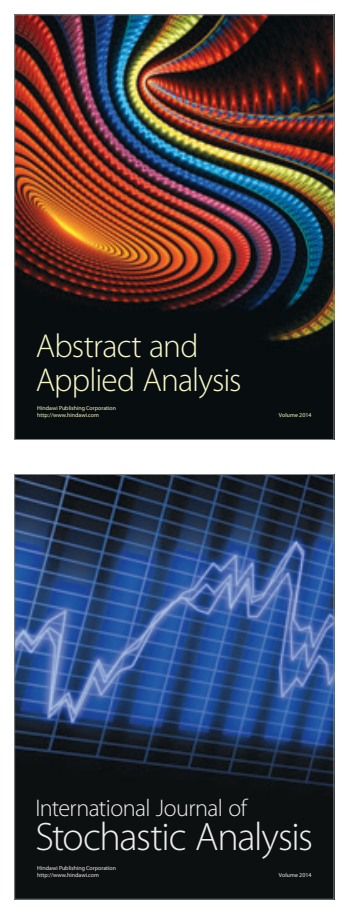

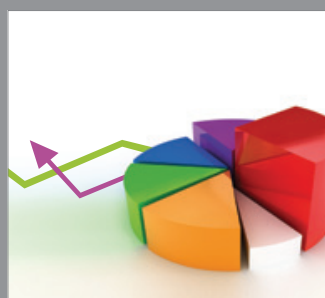

ournal of

Probability and Statistics

Promensencen
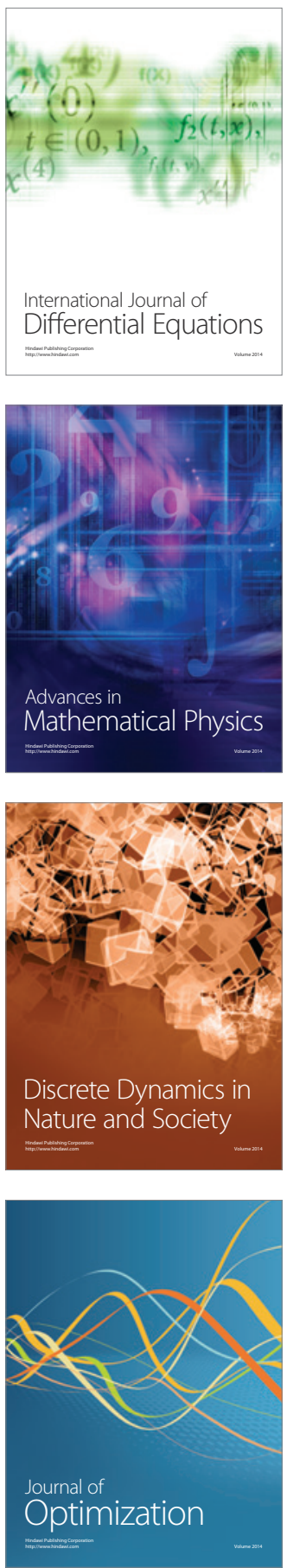